\title{
Median-Difference Correntropy for DOA under the Impulsive Noise Environment
}

\author{
Fuqiang Ma, ${ }^{1,2,3}$ Jie He $\mathbb{D}^{1,2,3}$ and Xiaotong Zhang $\mathbb{D D}^{1,2,3}$ \\ ${ }^{1}$ Beijing Advanced Innovation Center for Materials Genome Engineering, University of Science and Technology Beijing, \\ Beijing 100083, China \\ ${ }^{2}$ Department of Computer Science and Technology, University of Science and Technology Beijing, Beijing 100083, China \\ ${ }^{3}$ Beijing Key Laboratory of Knowledge Engineering for Materials Science, Beijing 100083, China \\ Correspondence should be addressed to Xiaotong Zhang; zxt@ies.ustb.edu.cn
}

Received 25 May 2019; Revised 21 August 2019; Accepted 16 September 2019; Published 3 October 2019

Guest Editor: Peio Lopez-Iturri

Copyright (C) 2019 Fuqiang Ma et al. This is an open access article distributed under the Creative Commons Attribution License, which permits unrestricted use, distribution, and reproduction in any medium, provided the original work is properly cited.

\begin{abstract}
The source localization using direction of arrival (DOA) of target is an important research in the field of Internet of Things (IoTs). However, correntropy suffers the performance degradation for direction of arrival when the two signals contain the similar impulsive noise, which cannot be detected by the difference between two signals. This paper proposes a new correntropy, called the median-difference correntropy, which combines the generalized correntropy and the median difference. The median difference is defined as the deviation between the sampling value and the median of the signal, and it intuitively reflects the abnormality of impulsive noise. Then, the median difference is combined with the generalized correntropy to form a new weighting factor that can effectively suppress the amplitude level of impulsive noise. To improve the robustness of the algorithm, an adaptive kernel size is also integrated into the weighting factor to obtain the optimal local feature. The influence of adaptive kernel sizes on the proposed algorithm is simulated, and the comparison between three typical direction-of-arrival estimation algorithms is conducted. The results show that the accuracy of the median-difference correntropy is significantly superior to the correntropybased correlation and the phased fractional lower-order moment for a wide range of alpha-stable distribution noise environments.
\end{abstract}

\section{Introduction}

The source localization using direction of arrival (DOA) of target is an important research in the field of Internet of Things (IoTs). The direction-of-arrival (DOA) approaches based on acoustics have many applications including radar, sonar, seismic exploration, navigation, and sound source tracking [1-3]. DOA estimation is usually regarded as a problem of signal matching, and the performance is significantly influenced by noise. A majority of existing DOA estimations are based on the concept that noise follows Gaussian distribution $[4,5]$. Since the Gaussian process has second-order and higher-order statistics, the traditional DOA algorithms can easily evaluate the signal characteristics according to second-order statistics [6]. The multiple signal classification (MUSIC) algorithm [7, 8] and estimation method of signal parameters via rotational invariance techniques (ESPRIT) are the basic subspace algorithms which have good performance $[9,10]$. MUSIC is the representative of the noise subspace algorithm, and ESPRIT is the representative of the signal subspace algorithm.

Because of atmospheric noise, electromagnetic interference, sea clutter, car ignitions, and office equipment, the signal is corrupted by the extremely impulsive noise that exhibits irregularity in time domain. In addition, the probability density functions of impulsive noise decay with heavy tails and do not follow a common Gaussian distribution [11]. Therefore, alpha-stable distribution is usually used to define impulsive noise [12]. The conventional covariance matrix is calculated from the second-order statistics of the signal, which may be infinite when the data are corrupted by the extremely impulsive noise $[13,14]$. In addition, the conventional DOA algorithms cannot be decomposed into the signal subspace and the noise subspace 
with covariance matrix. Thus, it has become increasingly important to study DOA under the impulsive noise environment.

The relevant statistical algorithms mainly focus on the existence of statistics. The fractional lower-order statistics (FLOS) algorithms [15] exhibit a more desirable performance than the second-order statistics for alpha-stable distribution [16]. When the signal contains impulsive noise, the signal bears the fractional lower-order statistics. The FLOS algorithms employ the minimum dispersion (MD) criterion to suppress impulsive noise, such as the robust covariation in ROC-MUSIC [17], the fractional lower-order statistics- (FLOM-) based MUSIC, and the phased fractional lower-order moment (PFLOM). The FLOM algorithmbased MUSIC obtains a finite covariance by suppressing one of two cross-correlation signals when the characteristic exponent of alpha-stable distribution ranges from 1 to 2 [18]. The PFLOM algorithm gets the accurate DOA estimation with circular symmetrical signals, embedded in the additive impulsive noise [19]. However, investigators demonstrate that the performance of FLOM and PFLOM algorithms depends on the relationship between the parameter of fractional lower-order moment and the characteristic exponent of alpha-stable distribution. If the characteristic exponent is unknown, the performance of FLOM and PFLOM algorithms seriously decreases.

The correntropy criterion [20] is a relatively simple method that can measure the local similarity between two signals [21, 22]. Because it has the properties of M-estimation, the correntropy has been widely used in the impulsive noise environment [23]. Zhang et al. [24] investigated a narrowband model based on the generalized correntropy which is called the correntropy-based correlation (CRCO) in impulsive noise environment. The CRCO algorithm imposes a correntropy operator on the covariance matrix to depress impulsive noise. The generalized correntropy is suitably for dealing with the template matching between the received signal and the template signal [25]. Since DOA estimation is a matching problem between two signals, the generalized correntropy is incapable of measuring the difference between the outliers and fails to suppress impulsive noise. Thus, when data contain the similar impulsive noise, the cross-correlation of the generalized correntropy is infinite.

In order to solve the problem that correntropy cannot distinguish the similar impulsive noise, we propose a median-difference correntropy (MDCO) algorithm. The MDCO depending on the inner product of vectors measures the similarity of multidimensional properties from input space. A weighting factor of a median difference is defined and evaluates the similarity between the sample value and the median of the signal. The median difference intuitively reflects the abnormality of impulsive noise to guarantee that the autocorrelation is finite. Then, MDCO derives the weighting factor of the generalized correntropy from the correntropy criterion which suppresses the larger impulsive noise. Hence, the weighted covariance function of signal employs the generalized correntropy instead of second-order statistics. These two criteria map the signal from the low-dimensional space to an infinite-dimensional reproducing kernel Hilbert space (RKHS) with impulsive noise, thus including higher-order signal statistics. The adaptive kernel size is applied to the weighting factor for MDCO which can obtain the optimal local feature. At convergence, MDCO is unbiased and it is applicable to achieve CRLB under various parameters. The main work is summarized as follows:

(1) We propose a median-difference correntropy (MDCO) algorithm, which can effectively combine the generalized correntropy and the median difference to suppress impulsive noise

(2) To improve the robustness of MDCO, we also introduce a novel adaptive kernel size into the weighting factor of the generalized correntropy and the median difference

The rest of this paper is organized as follows: The problem model is defined with DOA in Section 2. In Section 3 , we present the median-difference correntropy (MDCO) for DOA estimation under impulsive noise environment. The performance evaluation is presented in Section 4. Finally, conclusions are drawn in Section 5.

\section{Problem Formulation}

It is considered that a uniform linear array of $M$ isotropic acoustic sensors receives the far-field signal generated by $P(M>P)$ narrowband sources. Then, Figure 1 illustrates the linear array model.

The first array sensor is a reference sensor, and the received signal of the $m$ th sensor can be expressed as

$$
x_{m}=\sum_{p=1}^{P} s_{p}(t) e^{-j 2 \pi f \tau_{m p}}+n_{m}(t),
$$

where $s_{p}(t)$ is the $p$ th signal at time $t, n_{m}(t)$ is the noise of the $m$ th sensor, $\tau_{m p}$ denotes the time delay of the $m$ th acoustics sensor relative to the reference sensor for the $p$ th signal, and $f$ is the center frequency of signal.

The received signal of acoustics can be expressed as

$$
\mathbf{X}(t)=\mathbf{A}(\theta) \mathbf{S}(t)+\mathbf{N}(t)
$$

where

$$
\begin{aligned}
\mathbf{X}(t) & =\left[x_{1}(t), x_{2}(t), \ldots, x_{M}(t)\right]^{T}, \\
\mathbf{A}(\boldsymbol{\theta}) & =\left[\mathbf{a}\left(\theta_{1}\right), \mathbf{a}\left(\theta_{2}\right), \ldots, \mathbf{a}\left(\theta_{P}\right)\right], \\
\mathbf{S}(t) & =\left[s_{1}(t), s_{2}(t), \ldots, s_{P}(t)\right]^{T}, \\
\mathbf{N}(t) & =\left[n_{1}(t), n_{2}(t), \ldots, n_{M}(t)\right]^{T},
\end{aligned}
$$

in which the superscript $[\bullet]^{T}$ represents transpose, $\mathbf{X}(t)$ is the $M \times 1$ vector of the signal received by the acoustic array sensors, $\mathbf{S}(t)$ is the $P \times 1$ vector of the acoustic source, $\mathbf{N}(t)$ is the $M \times 1$ vector of impulsive noise that follows alphastable distribution, and $\mathbf{A}(\boldsymbol{\theta})$ is the $M \times P$ array manifolds of the array sensors. There is an assumption that the number $M$ of the array elements is greater than the number $P$ of the acoustic sources. $\mathbf{a}\left(\theta_{p}\right)$ is the steering vector that can be expressed as 


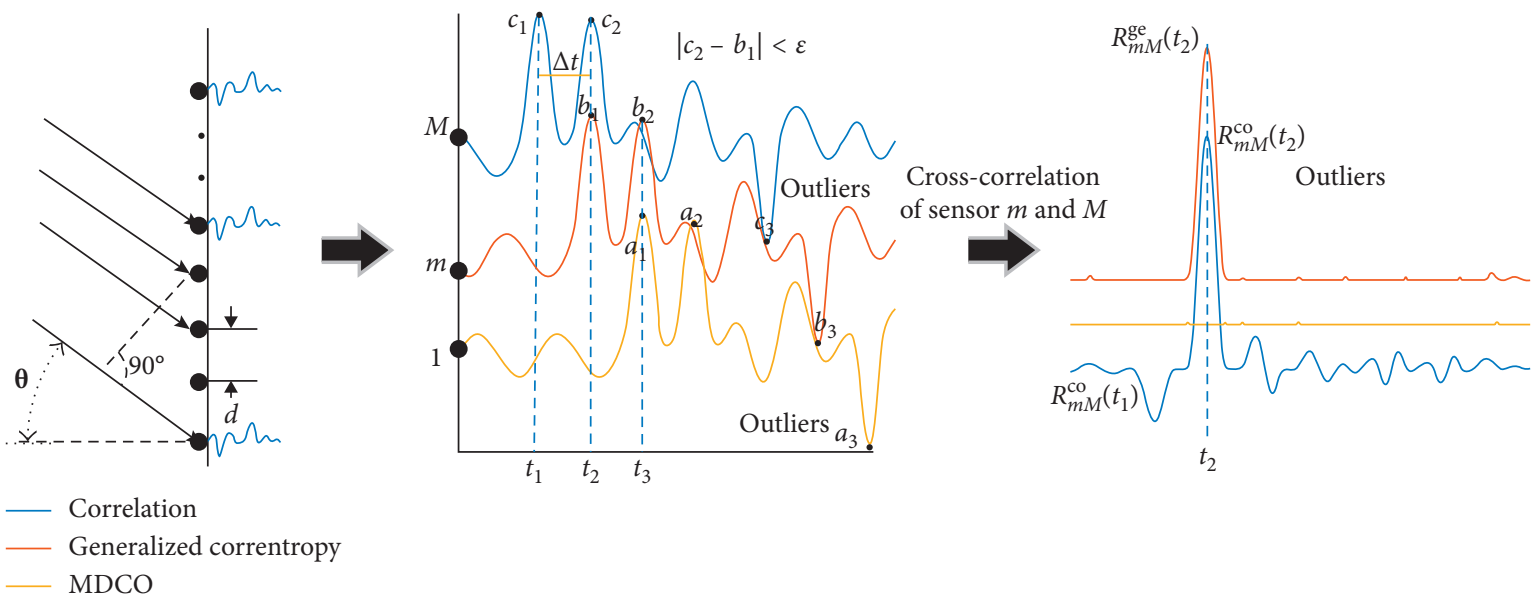

Figure 1: Model of the uniform linear array.

$$
\mathbf{a}\left(\theta_{p}\right)=\left[1, e^{-j 2 \pi f \tau_{2 p}}, \ldots, e^{-j 2 \pi f \tau_{M p}}\right]^{T} .
$$

Impulsive noise $\mathbf{N}(t)$ in (2) is usually defined as symmetric alpha-stable distribution. However, the probability density function of symmetric alpha-stable distribution cannot be expressed by a general expression [24]. Therefore, it is generally introduced by its characteristic function which can be expressed as

$$
\phi(t)=\exp \left\{-\gamma|t|^{\alpha}\right\}
$$

where $\alpha$ is the characteristic exponent of symmetric alphastable distribution whose range is $0<\alpha \leq 2$. Moreover, the smaller the $\alpha$ becomes, the more impulsive the nonGaussian noise will be. $\gamma$ is the dispersion.

Usually, the received data contain very little impulsive noise and can also be represented as

$$
x_{m}=\sum_{p=1}^{P} s_{p}(t) e^{-j 2 \pi f \tau_{m p}}+w_{m}(t)+e_{m}(t),
$$

where $e_{m}(t)$ denotes the noise without outliers, $w_{m}(t)$ denotes outliers with sparse characteristics, $\mathbf{W}(t)=\left[w_{1}(t)\right.$, $\left.w_{2}(t), \ldots, w_{M}(t)\right]^{T}$, and $\mathbf{E}(t)=\left[e_{1}(t), e_{2}(t), \ldots, e_{M}(t)\right]^{T}$. Meanwhile, $x^{\prime}=s+e$ denotes the received data without outliers. We can assume that the noise $e_{m}(t)$ follows Gaussian distribution. Then, the noise $n_{m}(t)=e_{m}(t)+$ $w_{m}(t)$ follows symmetric alpha-stable distribution. The purpose of our algorithm is to eliminate outliers $w_{m}(t)$.

Many DOA estimation algorithms usually use the covariance to represent the second-order statistics of the signal. The conventional covariance matrix can be given as

$$
\mathbf{R}=E\left[\mathbf{X}(t) \mathbf{X}(t)^{H}\right]=\mathbf{A}(\theta) \mathbf{R}_{s} \mathbf{A}^{H}(\theta)+\mathbf{R}_{n},
$$

where the superscript $[\bullet]^{H}$ represents transpose, the notation $E[\bullet]$ represents the expectation operation, and $\mathbf{R}_{s}$ and $\mathbf{R}_{n}$ are the covariance matrix of signal and noise, respectively. If the noise contains impulsive noise, the signal and noise cannot be completely orthogonal. The conventional DOA algorithms that describe signal with impulsive noise cannot be decomposed into the signal subspace and the noise subspace by the covariance matrix. Therefore, the covariance matrix (7) can also be represented as

$$
\begin{aligned}
\mathbf{R}=\mathbf{E}\left[\mathbf{X}(t) \mathbf{X}(t)^{H}\right] & =\mathbf{A}(\theta) \mathbf{R}_{s} \mathbf{A}^{H}(\theta)+\mathbf{R}_{e}+\mathbf{R}_{w}, \\
\mathbf{R}_{w} & =\mathbf{E} \mathbf{W}^{H}+\mathbf{W} \mathbf{E}^{H}+\mathbf{W} \mathbf{W}^{H},
\end{aligned}
$$

where $\mathbf{R}_{w}$ denotes the covariance term generated by outliers and the covariance $\mathbf{R}$ is dominated by impulsive noise when some elements of covariance $\mathbf{R}_{w}$ is much larger than the corresponding elements of $\mathbf{A}(\theta) \mathbf{R}_{s} \mathbf{A}^{H}(\theta)+\mathbf{R}_{e}$. Because natural noise and man-made noise often include outliers, some elements of matrix $\mathbf{R}_{w}$ may have a large value which gives rise to false DOA estimation. From Figure 1, we can see that the received data of the acoustic sensors suffer impulsive noise with large values $\left\{a_{1}, a_{2}, a_{3}, b_{1}, b_{2}\right.$, $\left.b_{3}, c_{1}, c_{2}, c_{3}\right\}$. For example, because data $b_{1}$ and $c_{2}$ which are located on the different sensors are both impulsive noise at the same time, the cross-correlation with a relatively large value is infinite:

$$
\begin{aligned}
R_{m M}^{\mathrm{co}}\left(t_{2}\right) & =Y Z=X_{m}\left(t_{2}\right) X_{M}\left(t_{2}\right) \\
& =X_{M}\left(t_{1}+\Delta t\right) X_{M}\left(t_{2}\right) e^{j 2 \pi f \Delta t}=b_{1} c_{2},
\end{aligned}
$$

where $Y$ and $Z$ are two sampling points of total snapshots and $R_{m M}^{\mathrm{co}}\left(t_{2}\right)$ represents the cross-correlation of data $b_{1}$ and $c_{2}$ at time $t_{2}$. Assuming that only one acoustic source impinges on the array, the signal $X_{m}\left(t_{2}\right)$ is a delayed signal of $X_{M}\left(t_{1}\right)$ with time $\Delta t$ when the signal $X_{M}\left(t_{1}\right)$ is received by the array sensor $M$ at time $t_{1}$. Furthermore, array sensors $m$ and $M$ both contain impulsive noise at time $t_{2}$. At this point, the noise covariance $R_{w}\left(t_{2}\right)$ is dominant so that the noise subspace would spread to the signal subspace, causing the characteristics of the signal to be covered. In addition, the autocorrelation of data $a_{1}, b_{1}$, and $c_{1}$ is also nonexistent.

The goal is to search for an efficient strategy of suppressing impulsive noise that makes it possible to

$$
\lim _{w_{m}(t) \longrightarrow \infty} \psi\left(\mathbf{R}_{w}\right) \approx 0,
$$

where $\psi\left(\mathbf{R}_{w}\right)$ indicates the operator of suppressing impulsive noise. In principle, as long as $\mathbf{R}_{w}$ provides a small 
contribution, we can accurately estimate DOA from the covariance.

\section{Median-Difference Correntropy (MDCO)}

In order to solve impulsive noise, the structure of this section is as follows: firstly, the median-difference correntropy (MDCO) is proposed. Next, we summarize some properties for MDCO. Finally, the application of MDCO for DOA estimation is designed.

Normally, if the signal follows Gaussian distribution, there is a $99.73 \%$ probability that results in $X^{\prime} \in\left(\mu^{\prime}-3 \sigma^{\prime}\right.$, $\left.\mu^{\prime}+3 \sigma^{\prime}\right)$, where $X^{\prime}$ is the collection of Gaussian distribution signal, $\mu^{\prime}$ is the mean value, and $\sigma^{\prime}$ is the standard deviation ( $\mu^{\prime}$ and $\sigma^{\prime}$ have no relationship to the similar parameters behind). If $X_{1}^{\prime}, X_{2}^{\prime} \in\left(\mu^{\prime}, \sigma^{\prime 2}\right)$, the $X_{1}^{\prime}-X_{2}^{\prime}$ is a zero-mean Gaussian process with variance $2 \sigma^{\prime 2}$. Therefore, there is a $99.73 \%$ probability of $X_{1}^{\prime}-X_{2}^{\prime} \in\left(-6 \sigma^{\prime}, 6 \sigma^{\prime}\right)$. At this point, we can simply set a threshold $\varepsilon$ to measure the similarity between the signals and set $\varepsilon=6 \sigma^{\prime}$.

Definition 1. For two variables $Y$ and $Z$ of total snapshots $\mathbf{X}$, where $Y, Z \in \mathbf{X}$, if $|Y-Z|<\varepsilon$, we say that the variable $Y$ is similar to $Z$.

As shown in Table 1, the similarity of the variables $Y$ and $Z$ is divided into four cases. If $Y, Z \in \mathbf{X}^{\prime},\left(\mathbf{X}^{\prime} \in \mathbf{X}\right)$, the variables $Y$ and $Z$ are considered normal values. But, if $Y, Z \in \mathbf{X}^{\prime}+\mathbf{W}$, where $\mathbf{X}^{\prime}+\mathbf{W} \in \mathbf{W}$, we consider the variables $Y$ and $Z$ to be the outliers containing impulsive noise. One of our goals is to suppress the three abnormal cases that contain impulsive noise.

3.1. Median-Difference Correntropy (MDCO). In this section, we present a median-difference correntropy (MDCO) algorithm for DOA with alpha-stable distribution. The correntropy is a new method for nonlinear and local optimal measurement of two random variables $Y$ and $Z$. It is defined as follows:

$$
V(Y, Z)=E_{Y Z}[\kappa(Y, Z)]=\iint \kappa(y, z) p_{Y, Z}(y, z) \mathrm{d} y \mathrm{~d} z
$$

where $\kappa(.,$.$) is a translation function of the shift-invariant$ Mercer kernel [26] and $p_{Y, Z}(.,$.$) is the joint probability$ density function of $Y$ and $Z$. In this paper, the Gaussian density function is used as kernel function. The Gaussian kernel creates a RKHS [26] with universal approximating capability. The Gaussian kernel is numerically stable and usually gets reasonable results. In this paper, we use a Gaussian kernel to suppress impulsive noise.

Assuming that the random variables $Y$ and $Z$ follow symmetric alpha-stable distribution whose characteristic exponent is $1<\alpha<2$, the median-difference correntropy (MDCO) can be considered as
TABLE 1: Comparison of similarity between two variables.

\begin{tabular}{cccc}
\hline Variables & $Y, Z \in \mathbf{X}^{\prime}$ & $\begin{array}{c}Y \in \mathbf{X}^{\prime} \\
Z \in \mathbf{X}^{\prime}+\mathbf{W} \\
|Y-Z|<\varepsilon\end{array}$ & $\begin{array}{c}Y \in \mathbf{X}^{\prime}+\mathbf{W} \\
Z \in \mathbf{X}^{\prime}+\mathbf{W}\end{array}$ \\
\hline Condition & $\mathrm{C} 1$ & $\mathrm{C} 2$ & $\mathrm{C} 3$ \\
Similarity & Yes & No & No \\
\hline$R_{\mathrm{MDCO}}=$ & $E\left[\exp \left(-\frac{|| Y|-B|^{2}}{2 \sigma^{2}}\right) \exp \left(-\frac{|| Z|-B|^{2}}{2 \sigma^{2}}\right)\right.$ \\
& $\left.\cdot \exp \left(-\frac{|Y-Z|^{2}}{2 \sigma^{2}}\right) Y Z\right]$,
\end{tabular}

where $\sigma$ is the kernel size of Gaussian kernel. The variable $B$ is the average of $l_{1}$ - norm of the preprocessing data which can approximately represent the median of the received data.

Because impulsive noise contained in the signal is sparse, the variable $B$ can effectively weigh the amplitude of the signal. The variables $|Y|-B$ and $|Z|-B$ reflect the median difference to which the received signal deviates from the median $B$. Therefore, the variables $|Y|-B$ and $|Z|-B$ are called the weighting factors of the median difference. The correntropy $Y-Z$ measures the similarity between the signals and is called the weighting factor of the generalized correntropy. The median difference evaluates the deviation between the sampling value and the median of the signal, and it intuitively reflects the abnormality of impulsive noise. Then, the median difference is combined with the generalized correntropy to form a new weighting factor, which can effectively suppress the amplitude level of impulsive noise. Then, the median difference and the generalized correntropy are combined with the traditional covariance, and a novel generalized weighted covariance is obtained.

To simplify (12), the median differences $|Y|-B$ and $|Z|-$ $B$ can be combined. The variable $|Y|+|Z|-2 B$ reflects the deviation degree of $|Y|+|Z|$. The relaxation of (12) can be expressed as

$$
R_{\mathrm{MDCO}}=E\left[\exp \left(-\frac{|| Y|+| Z|-2 B|^{2}}{2 \sigma_{1}^{2}}\right) \exp \left(-\frac{|Y-Z|^{2}}{2 \sigma_{2}^{2}}\right) Y Z\right] \text {, }
$$

where $|Y|$ and $|Z|$ are the absolute value of the signal and $\sigma_{1}$ and $\sigma_{2}$ are the kernel sizes that control the scale of the metric.

The short-time energy method is a common method to detect impulsive noise in time domain [27]. We use a shorttime average energy method to preprocess the received data. However, the data processed by the short-time average energy method does not directly be used as the input of MDCO. The short-time average energy indicates that the energy of the signal is average in a short segment. The shorttime average energy in the $k$ th segment can be expressed as $E_{k}$ :

$$
E_{k}=\frac{\sum_{i=k}^{k+I-1}(X(i))^{2}}{I}
$$


TABLE 2: Comparison of performance between CRCO and MDCO.

\begin{tabular}{lcccc}
\hline Variables & $Y, Z \in \mathbf{X}^{\prime},|Y-Z|<\varepsilon$ & $Y \in \mathbf{X}^{\prime}, Z \in \mathbf{X}^{\prime}+\mathbf{W},|Y-Z| \gg \varepsilon$ & \multicolumn{2}{c}{$Y \in \mathbf{X}^{\prime}+\mathbf{W}, Z \in \mathbf{X}^{\prime}+\mathbf{W}$} \\
& $\mathrm{C} 1$ & $\mathrm{C} 2$ & $|Y-Z| \gg \varepsilon$ & $\mathrm{C} 3$ \\
Condition & Success & Success & S4 \\
MDCO & & Success & Success \\
\hline
\end{tabular}

where $X(i)$ is the sampling sequence of the original signal. By the formula above, we can obtain the average energy with $I$ data points.

If the signal energy is much larger than the short-time average energy, the preprocessing signal $X_{\text {pre }}$ can be represented as

$$
X_{\text {pre }}(i)= \begin{cases}0, & E\left[(X(i))^{2}\right]>E_{k}, \\ X(i), & \text { others. }\end{cases}
$$

Assuming that $N_{0}$ is the sum of $E\left[(X(i))^{2}\right]>E_{k}$ in total snapshots. Therefore, in this study, $B$ can be expressed as

$$
B=\frac{\left\|\mathbf{X}_{\mathrm{pre}}\right\|_{1}}{N-N_{0}}
$$

where $N$ is the total sampling size of snapshots. The signal with impulsive noise does not participate in calculating the median of the signal. Therefore, for a majority of data, the median $B$ is less than $\varepsilon / 2$ due to preprocessing.

The key to the median-difference correntropy is that the kernel size can work in the confidence interval to eliminate impulsive noise. The kernel size is usually related to the dispersion coefficient $\gamma$ by considering the local feature of the signal. We take a modified Sigmoid function to make the kernel size adaptive $[24,28-30]$. Then, kernel sizes $\sigma_{1}$ and $\sigma_{2}$ can be expressed as

$$
\begin{aligned}
& \sigma_{1}^{2}=\frac{g_{1}}{1+\exp \left(-h_{1}(|| Y|+| Z|-2 B|)\right)} \cdot \gamma, \\
& \sigma_{2}^{2}=\frac{g_{2}}{1+\exp \left(-h_{2}(|Y-Z|)\right)} \cdot \gamma,
\end{aligned}
$$

where $g_{1}$ and $g_{2}$ indicate the scale of the modified Sigmoid function and $h_{1}$ and $h_{2}$ control the monotonicity of the modified Sigmoid function [28]. The shrinkage direction and rate are determined by $h_{1}$ and $h_{2}$, respectively.

The adaptive median-difference correntropy provides a new metric criterion for impulsive noise. And the M-estimators of the autocorrelation and cross-correlation of random variables $Y$ and $Z$ are in existence associated with the generalized correntropy and median difference.

In order to prove the effectiveness of MDCO in theory, five crucial properties of MDCO are listed as follows:

Property 1 (C1). When the noise follows temporally stationary zero-mean white Gaussian processes, MDCO is reduced to traditional second-order statistics, and the performance of $\mathrm{MDCO}$ is equal to traditional signal subspace algorithms (see Appendix A for this proof).
Property 2 (C2). If the variable $Y$ is an outlier rather than $Z$, the MDCO can eliminate impulsive noise. $R_{w}(t)$ of MDCO approaches zero; that is to say, $R_{\mathrm{MDCO}}$ is close to zero at this point (see Appendix B for this proof).

Property 3 (C3, C4). When the variables $Y$ and $Z$ both contain impulsive noise, $R_{w}(t)$ and $R_{\mathrm{MDCO}}$ both approach zero (see Appendix $C$ for this proof).

Property 4. When the variables $Y$ and $Z$ both contain impulsive noise, the MDCO is more effective than the CRCO for noise suppression (see Appendix D for this proof).

Property 5. Assuming that the random variables $Y$ and $Z$ follow the symmetric alpha-stable distribution, $R_{\mathrm{MDCO}}$ is bounded and the MDCO has the generalized correntropy statistics (see Appendix E for this proof).

Because of the abovementioned properties, the generalized weighted covariance $R_{\mathrm{MDCO}}$ has finite autocorrelation and cross-correlation at all moments. When the sampling data contain impulsive noise, $R_{w}(t)$ of MDCO approaches zero; that is to say, $R_{\mathrm{MDCO}}$ is close to zero at this point. If two signals contain the different degrees of impulsive noise at the same time, MDCO can induce a very small weighted covariance by means of the median difference operator and then obtain a convergent covariance matrix. As shown in Table 2, the performances of the MDCO is listed for different noise cases. In summary, the MDCO has reliably good performance in all cases.

\section{Performance Simulations}

In this experiment, we compare the MDCO-MUSIC to FLOM-MUSIC, PFLOM-MUSIC, and CRCO-MUSIC and test the performance of the proposed MDCO-MUSIC under different parameter conditions. In our experiment, we evaluate the performance of the algorithm from five aspects, namely, various kernel sizes of MDCO-MUSIC, GSNR, snapshots of sampling, different characteristic exponents of alpha-stable distribution, and angular separation of two direction angles.

The resolution probability can well define the performance of the four algorithms. We use a popular resolution criterion to measure the spatial spectrum which can be given as

$$
E\left[P\left(\theta_{m}\right)\right]<E\left[\frac{P\left(\theta_{1}\right)+P\left(\theta_{2}\right)}{2}\right],
$$

where $\theta_{1}$ and $\theta_{2}$ are the two independent direction angles, $\theta_{m}=\left(\theta_{1}+\theta_{2}\right) / 2$ is the midangle between them, and $P(\theta)$ is 


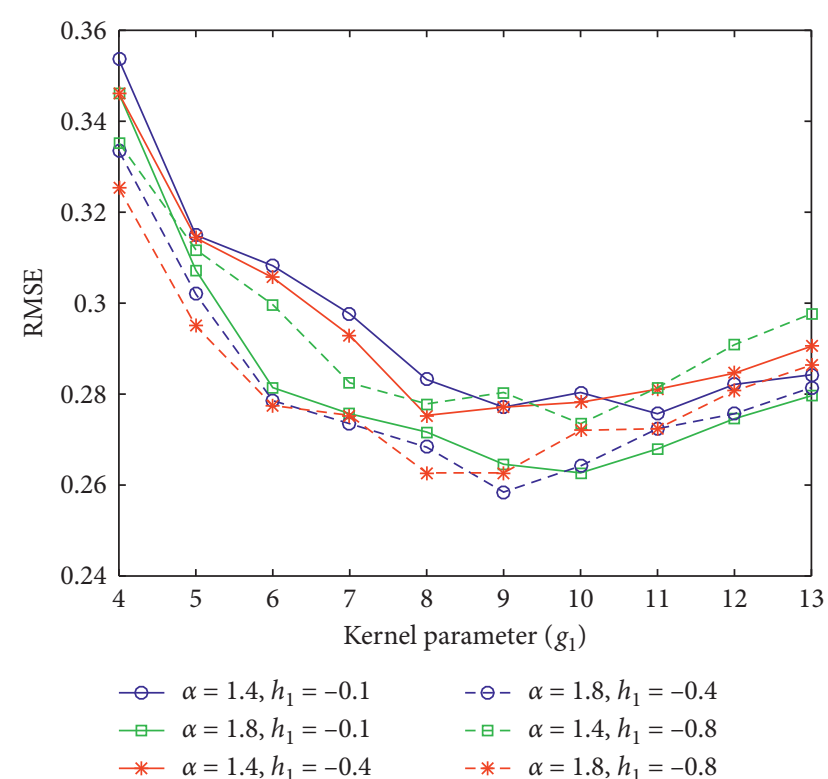

FIgURE 2: Performance comparison of the proposed algorithm versus kernel parameter $g_{1}$.

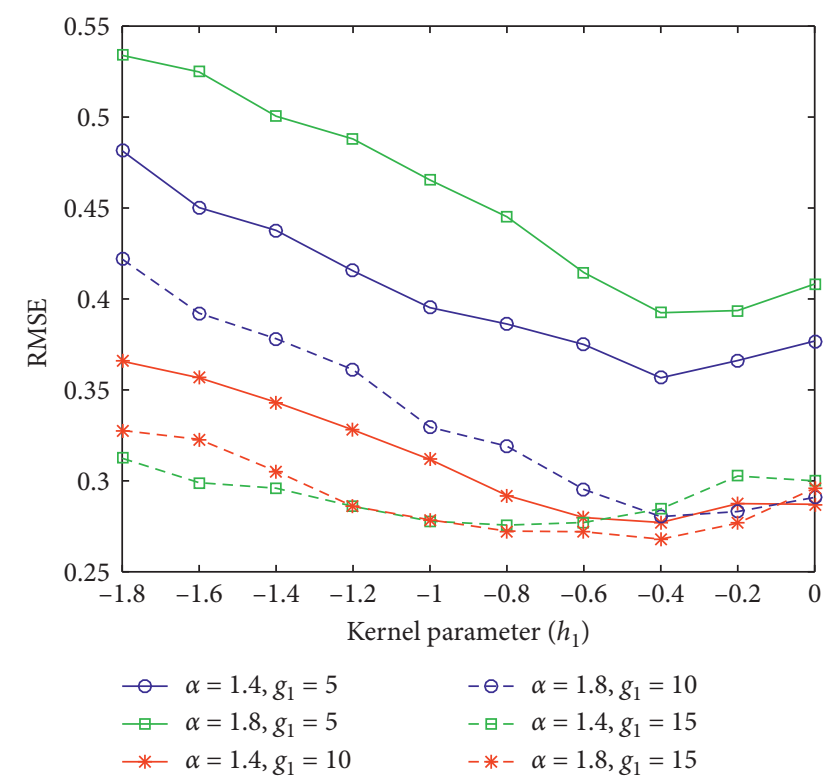

FIgURE 3: Performance comparison of the proposed algorithm versus kernel parameter $h_{1}$.

the spatial spectrum. The two direction angles are resolvable if the result on the left of the equation is smaller than that on the right; otherwise, the two direction angles are not resolvable.

RMSE is the deviation criterion between the observation and the true value and can be given by

$$
\mathrm{RMSE}=\sqrt{\frac{\sum_{l=1}^{L}\left(\hat{\theta}_{1}(l)-\theta_{1}\right)^{2}+\sum_{l=1}^{L}\left(\hat{\theta}_{2}(l)-\theta_{2}\right)^{2}}{2 L}},
$$

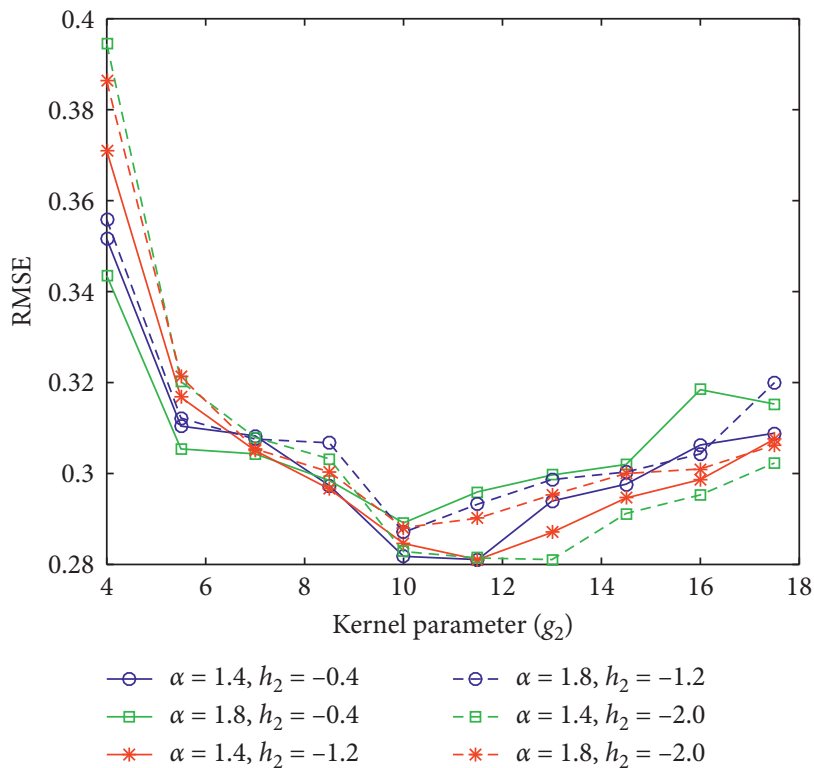

FIgURE 4: Performance comparison of the proposed algorithm versus kernel parameter $g_{2}$.

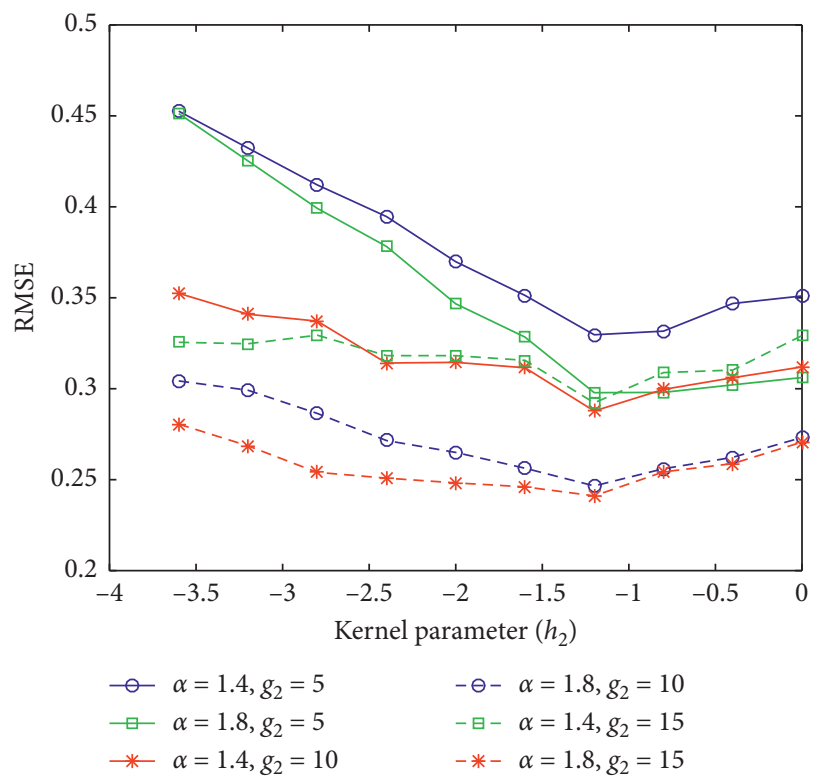

FIgURE 5: Performance comparison of the proposed algorithm versus kernel parameter $h_{2}$.

where $L$ represents the total number of MC run and $\widehat{\theta}_{1}(l)$ and $\widehat{\theta}_{2}(l)$ are estimations of $\theta_{1}$ and $\theta_{2}$ in the $l$ th $\mathrm{MC}$ run, respectively.

Kozick and Sadler has come to a closed-form expression of CRLB for the impulsive noise [31], which can be expressed as

$$
\mathrm{CRLB}=\frac{1}{I_{c}} \sum_{1}^{L} \operatorname{Re}\left\{\mathbf{S}_{d}^{H}(t) \mathbf{D}^{H}\left[\mathbf{I}-\mathbf{A}\left(\mathbf{A}^{H} \mathbf{A}\right)^{-1} \mathbf{A}^{H}\right] \mathbf{D S}_{d}(t)\right\},
$$




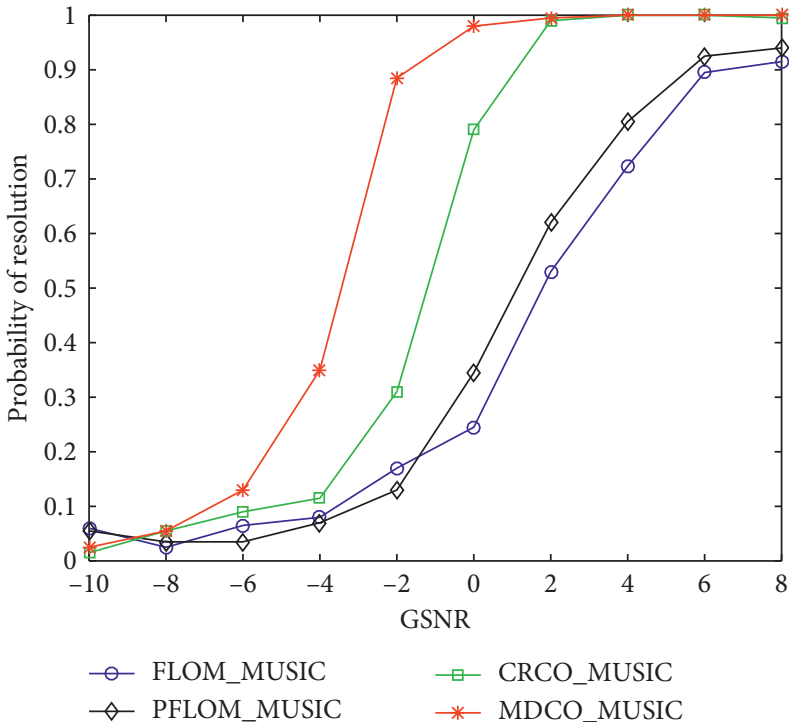

(a)

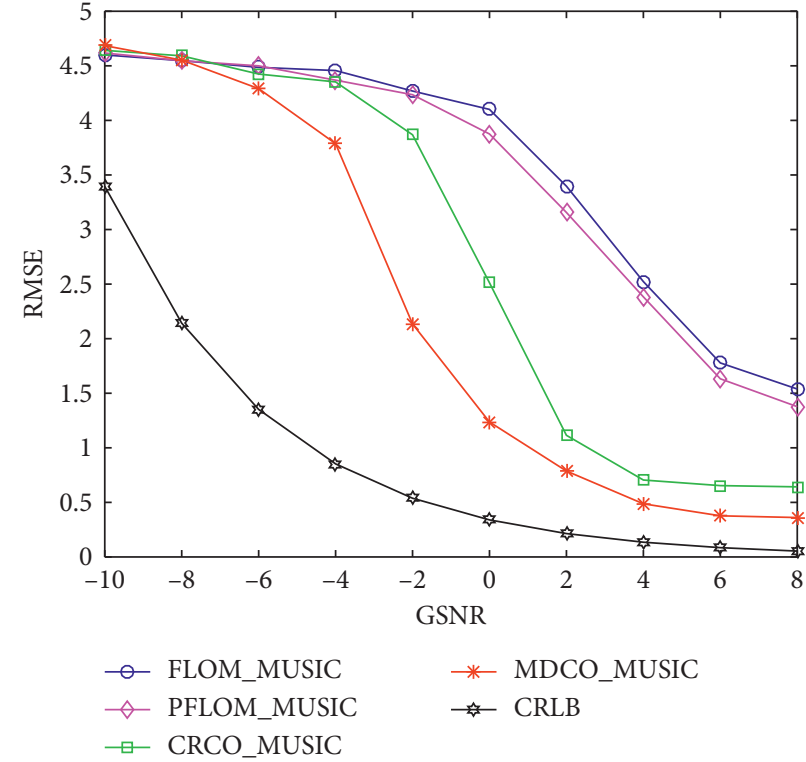

(b)

FIgURE 6: Performance comparison of four algorithms versus GSNR: (a) probability resolution of four algorithms; (b) RMSE of four algorithms.

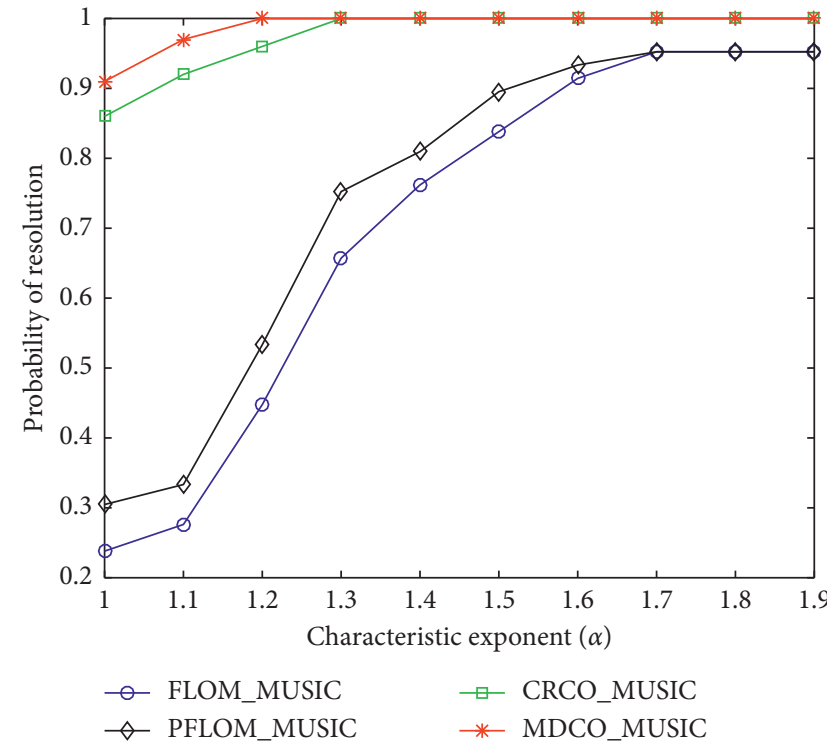

(a)

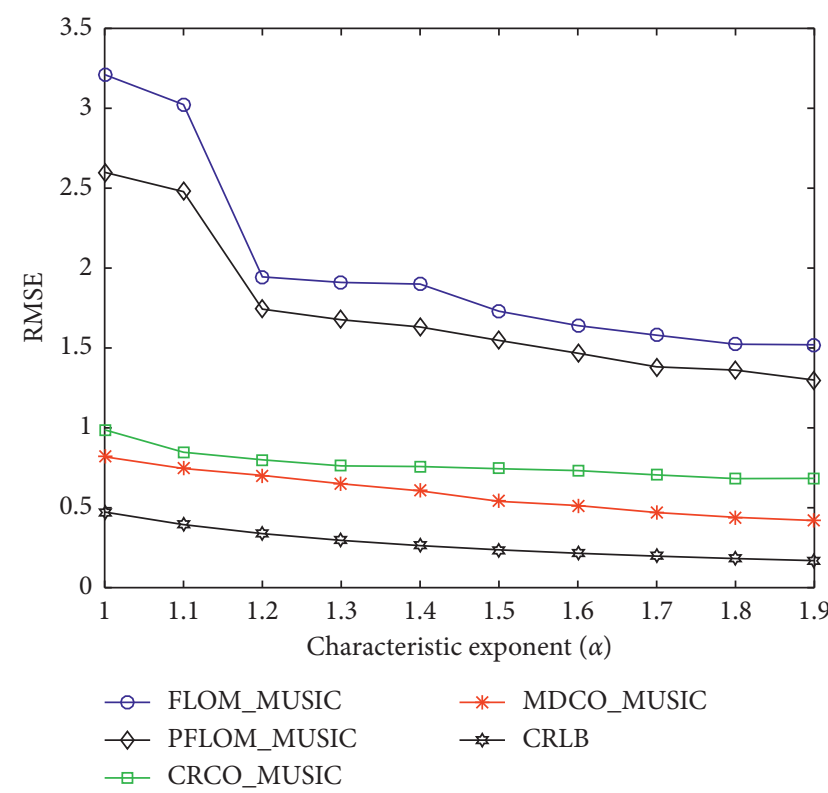

(b)

FIgURE 7: Performance comparison of four algorithms versus $\alpha$ values: (a) probability resolution of four algorithms; (b) RMSE of four algorithms.

where $\mathbf{S}_{d}(t)=\operatorname{diag}\left\{s_{1}(t), s_{2}(t), \ldots, s_{P}(t)\right\}$ is a diagonal matrix of the received signal, the subscript $\boldsymbol{\theta}$ of $\mathbf{A}$ is omitted from $\mathbf{A}(\boldsymbol{\theta}), \mathbf{D}=\left[\partial \mathbf{a}\left(\theta_{1}\right) / \partial \theta_{1}, \ldots, \partial \mathbf{a}\left(\theta_{P}\right) / \partial \theta_{P}\right]$ is the differential of the array manifolds $\mathbf{A}, \operatorname{Re}\{\cdot\}$ is the real part, and the coefficient $I_{c}$ can be expressed as $I_{c}=\int_{0}^{\infty}\left(\left(f^{\prime}(x)\right)^{2} /\right.$ $f(x)) x \mathrm{~d} x$, in which $x=|e|$ is the modulus of the impulsive noise and $f(x)$ is probability density function of $x$. Note that $I_{1}=1 / 2 \gamma$ with $\alpha=1$ and $I_{2}=3 / 5 \gamma^{2}$ with $\alpha=2$. For simplicity, the coefficient $I_{c}$ for $1<\alpha<2$ can be approximated by first-order linear interpolation with $I_{1}$ and $I_{2}$ [32].

4.1. Experimental Setup. The linear array is set to $M=6$ omnidirectional acoustic sensors which are placed in a half of the wavelength at the center frequency of the signal. We consider the case that the number of narrowband sources is 


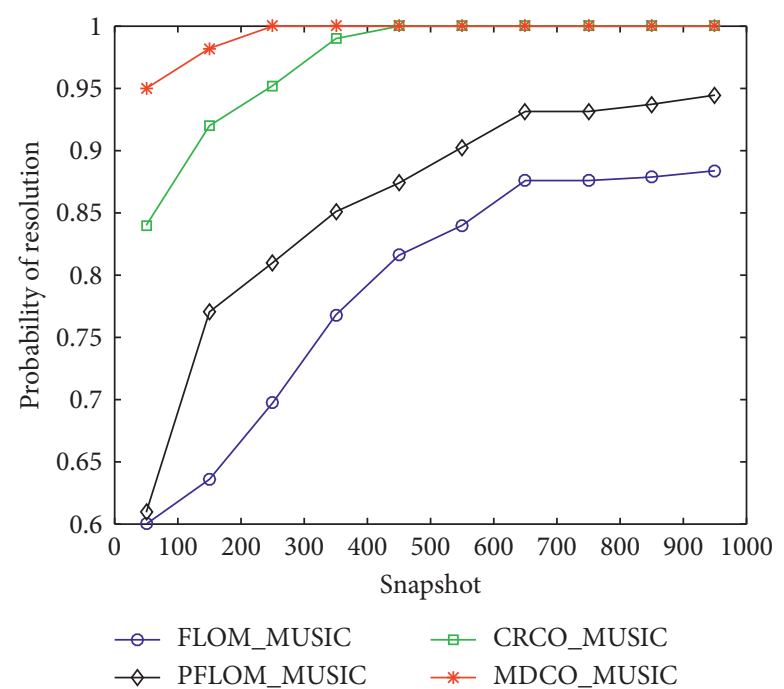

(a)
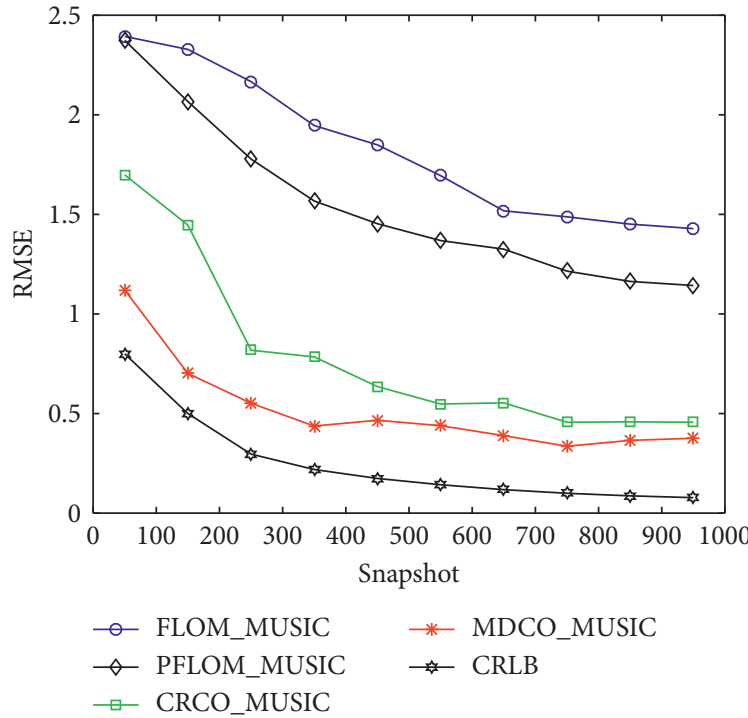

(b)

FIgURE 8: Performance comparison of four algorithms versus snapshot values: (a) probability resolution of four algorithms; (b) RMSE of four algorithms.

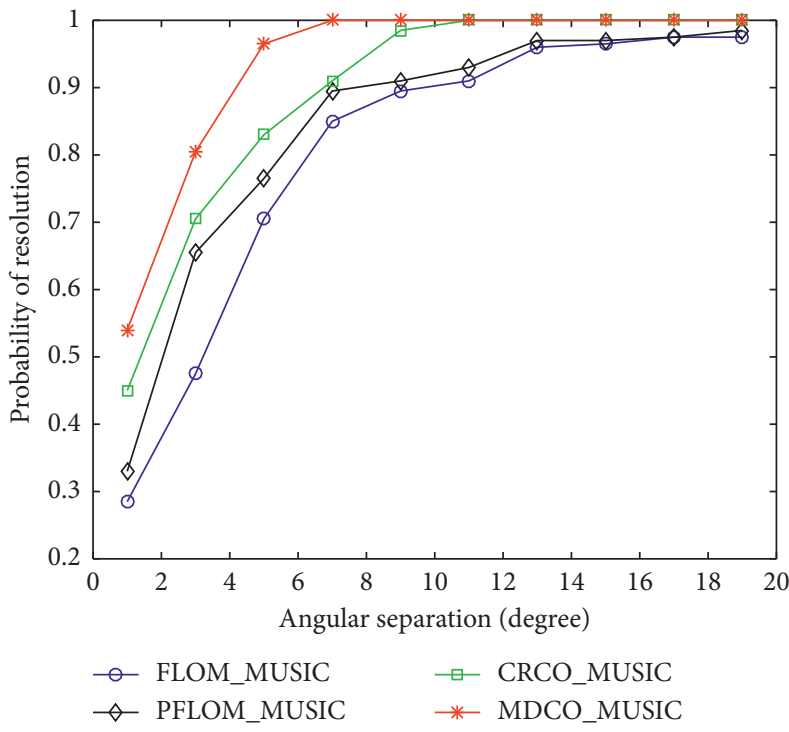

(a)

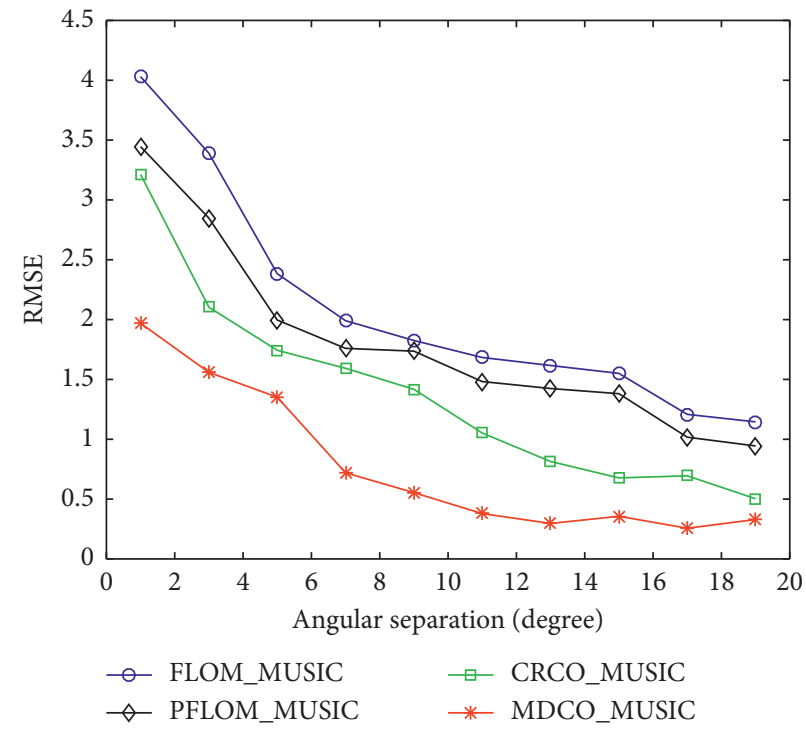

(b)

FIGURE 9: Performance comparison of four algorithms versus angular separation: (a) probability resolution of four algorithms; (b) RMSE of four algorithms.

two which follow temporally stationary zero-mean white Gaussian processes. The central frequency of signal is set to $f=100 \mathrm{~Hz}$. In order to distinguish the signal, the power of each signal is different. The symmetric alpha-stable distribution is used to model impulsive noise with the characteristic exponent $\alpha=1.4$ and dispersion $\gamma=1$. The GSNR is set to GSNR $=5 \mathrm{~dB}$. The sampling frequency of each array element is $F_{s}=240 \mathrm{~Hz}$. All experiments are carried out through two directions of arrival associated with $\theta_{1}=-10^{\circ}$ and $\theta_{2}=15^{\circ}$. The snapshots are snaps $=512$. Assume that the parameter $p$ of the fractional lower-order statistics is equal to 1.1 in FLOM-MUSIC and PFLOM-MUSIC algorithms. In every experiment, 500 Monte Carlo (MC) runs are performed. Furthermore, we compare the resolution probability and the root-mean-square error (RMSE) between the different algorithms.

4.2. Kernel Parameters of MDCO. In this experiment, we test the performance of MDCO-MUSIC with respect to the different kernel parameters of the weighting factors for the separate characteristic exponents $\alpha=1.4$ and $\alpha=1.8$. In 
order to eliminate impulsive noise, it is necessary to satisfy the requirement that the large local weighting factor of the median difference and generalized correntropy associate with the small kernel sizes $\sigma_{1}^{2}$ and $\sigma_{2}^{2}$; that is, $h_{1}$ and $h_{2}$ must be less than zero. Because the weighting factor of the median difference and generalized correntropy are independent parameters, the performance can be analyzed separately. Figures 2 and 3 show that the appropriate $g_{1}$ and $h_{1}$ for the median difference can effectively suppress impulsive noise and evidently decrease RMSE. From Figure 2, we can see that $g_{1} \in[8,11]$ would get the best result with three different values of $h_{1}$. The convergence of $g_{1}$ is not affected by $h_{1}$. Empirically, it is revealed that impulsive noise can be effectively alleviated in Figure 3, when $h_{1} \in[-0.5,-0.3]$.

Figures 4 and 5 illustrate the robustness of MDCOMUSIC in terms of the appropriate $g_{2}$ and $h_{2}$ for the generalized correntropy. MDCO-MUSIC gains the optimal performance when the kernel parameter $g_{2}$ varies from 9 to 12. Figure 5 shows that RMSE gains a more significant decrease if $h_{2} \in[-1.4,-1]$. Usually, it is a common method that the optimal kernel parameters are obtained by experiments. Therefore, we set $g_{1}=10, h_{1}=-0.4, g_{2}=10$, and $h_{2}=-1.2$ in other experiments.

\subsection{Performance of Different Algorithms}

4.3.1. Performance Comparison versus GSNR. Figure 6 analyzes the performance of MDCO-MUSIC, FLOM-MUSIC, PFLOM-MUSIC, and CRCO-MUSIC under the average GSNR from $-10 \mathrm{~dB}$ to $8 \mathrm{~dB}$. We can derive the conclusion that MDCO-MUSIC outperforms FLOM-MUSIC, PFLOM-MUSIC, and CRCO-MUSIC algorithms both in resolution probability and in RMSE. The MDCO-MUSIC algorithm yields particularly robust estimation to impulsive noise and can accurately estimate the DOA when the GSNR is $2 \mathrm{~dB}$. The RMSE of MDCO-MUSIC is much smaller than that of FLOMMUSIC and PFLOM-MUSIC. It is clear from Figure 6 that that the performance of MDCO coincides with the CRLB at high SNR.

4.3.2. Performance Comparison versus Characteristic Exponent of Alpha-Stable Distribution. In this simulation, we aim at evaluating the performance of the characteristic exponent on the basis of probability of resolution and RMSE. The characteristic exponent of the symmetric alpha-stable distribution ranges from 1 to 2 . Figure 7 shows that the performance of MDCO-MUSIC outperforms the FLOMMUSIC, PFLOM-MUSIC, and CRCO-MUSIC both in resolution probability and in RMSE. Moreover, the smaller the $\alpha$ becomes, the worse the performance of FLOM-MUSIC and PFLOM-MUSIC will be. Another observation is that it is beneficial to employ MDCO-MUSIC rather than PFLOMMUSIC if the source signal is not circularly symmetrical. Furthermore, when $\alpha$ approaches 1, FLOM-MUSIC and PFLOM-MUSIC gain a large RMSE if the parameters of the fractional lower-order statistics cannot automatically adapt. The result shows that the accuracy of the PFLOM-MUSIC algorithm is influenced by the relationship of the FLOM parameter $p$ and the characteristic exponent $\alpha$. Because of the contribution of two weighting factors, MDCO-MUSIC is not affected by the variety of characteristic exponent and can effectively estimate the DOA in any case.

\subsubsection{Performance Comparison versus Snapshots of} Sampling. In this simulation, the performance of MDCOMUSIC, FLOM-MUSIC, PFLOM-MUSIC, and CRCOMUSIC about snapshots is tested under symmetric alpha-stable distribution. Considering that the snapshots start at 50 and end at about 1000. Figure 8 illustrates that MDCO-MUSIC gains a more significant enhancement in resolution probability with the increasing number of snapshots and a more evident decrease in RMSE compared to the other three algorithms. Consequently, MDCOMUSIC can work effectively for a small number of snapshots.

\subsubsection{Performance Comparison versus Angular Separation.} Figure 9 depicts the performance of four algorithms when the direction angle of the second signal is gradually away from that of the first angle. The first direction of arrival is $\theta_{1}=-10^{\circ}$. As expected, the MDCO-MUSIC can effectively estimate the two directions of arrival with $7^{\circ}$ angle separation. Furthermore, MDCO-MUSIC requires a smaller angle separation threshold than FLOM-MUSIC, PFLOMMUSIC, and CRCO-MUSIC associated with a fixed probability of resolution, and then MDCO-MUSIC gains a lower RMSE than other algorithms associated with a fixed angular separation threshold.

Through the RMSE curve in the above experiment, MDCO can effectively converge to a stable trend under different parameters, which ensure the boundedness and improve the robustness of the algorithm.

\section{Conclusion}

The issue of DOA in the presence of the abnormal similarity is solved through the adaptive median-difference correntropy. The MDCO combines the weighting factor of the median difference and generalized correntropy to suppress impulsive noise. The median difference evaluates the deviation between the sampling value and the median of the signal, and it intuitively reflects the abnormality of impulsive noise. The generalized correntropy measures the similarity between the two signals. These two weighting factors map the signal to an infinite-dimensional space with impulsive noise, thus including much statistical information. By controlling the adaptive kernel size, MDCO can effectively deal with DOA. We also prove that the MDCO satisfies some robust properties and applies the MDCO to DOA estimation combined with MUSIC. Experimental results illustrate that the MDCO-MUSIC is more robust than the FLOM-MUSIC, PFLOM-MUSIC, and CRCO-MUSIC at a much lower GSNR and in the extremely impulsive noise environment. 


\section{Appendix}

\section{A. Proof of Property 1}

In (12), the weighting factors can be represented as

$$
C=\exp \left(-\frac{|| Y|-B|^{2}}{2 \sigma^{2}}\right) \exp \left(-\frac{|| Z|-B|^{2}}{2 \sigma^{2}}\right) \exp \left(-\frac{|Y-Z|^{2}}{2 \sigma^{2}}\right) .
$$

When the noise does not contain impulsive noise, it can be obtained:

$$
\begin{aligned}
& 0<|| Y|-B|<\varepsilon, \\
& 0<|| Z|-B|<\varepsilon, \\
& 0<|Y-Z|<\varepsilon .
\end{aligned}
$$
into

The constraint of the weight factor $C$ can be translated

$$
\exp \left(-\frac{\varepsilon^{2}}{2 \sigma^{2}}\right)^{3}<C<1
$$

At this point, a small enough positive number $\xi$ can always be found so that the following formula is established:

$$
\frac{\varepsilon^{2}}{2 \sigma^{2}}<\xi
$$

Thus, it is easy to arrive at $C \approx 1$. In other words, the authors can get

$$
R_{\mathrm{MDCO}} \approx R=E[Y Z] \text {, }
$$

which means that the MDCO is reduced to the traditional second-order statistics algorithm when the noise follows Gaussian distribution.

\section{B. Proof of Property 2}

If the variable $Y$ is an outlier rather than $Z$, that is, $Y \gg Y^{\prime}$ and $Z=Z^{\prime}$, then $|Y-Z| \gg \varepsilon,|| Y|-B| \gg \varepsilon$, and ||$Z|-B|<\varepsilon$. Therefore, the weighting factor of generalized correntropy $Y-Z$ and median difference $|Y|-B$ is very small which can effectively suppress outlier $Y$. Let $R_{\mathrm{MDCO}}$ be expressed as

$$
\begin{aligned}
R_{\mathrm{MDCO}} & \approx E\left[\exp \left(-\frac{\left|W_{Y}\right|^{2}}{2 \sigma^{2}}\right) \exp \left(-\frac{|\varepsilon|^{2}}{2 \sigma^{2}}\right) \exp \left(-\frac{\left|W_{Y}\right|^{2}}{2 \sigma^{2}}\right) Y Z\right] \\
& \approx E[0 \times 1 \times 0 \times Y Z]=0,
\end{aligned}
$$

where $R_{\mathrm{MDCO}}$ results in a small value that is close to zero.

\section{Proof of Property 3}

If the variables $Y$ and $Z$ are signals with impulsive noise, the authors can obtain ||$Y|-B| \gg \varepsilon$ and ||$Z|-B| \gg \varepsilon$. If $W_{Y} \approx W_{Z}$, the variable $Y$ is similar to $Z$ and $|Y-Z|<\varepsilon$; otherwise, $|Y-Z|>\varepsilon$. Let us discuss the two cases together, and $R_{\mathrm{MDCO}}$ can be expressed as

$$
\begin{aligned}
R_{\mathrm{MDCO}}= & E\left[\exp \left(-\frac{\left|W_{Y}\right|^{2}}{2 \sigma^{2}}\right) \exp \left(-\frac{\left|W_{Z}\right|^{2}}{2 \sigma^{2}}\right)\right. \\
& \left.\cdot \exp \left(-\frac{\left|W_{Y}-W_{Z}\right|^{2}}{2 \sigma^{2}}\right) Y Z\right] \\
\approx & E\left[0 \times 0 \times \exp \left(-\frac{\left|W_{Y}-W_{Z}\right|^{2}}{2 \sigma^{2}}\right) \times Y Z\right]=0 .
\end{aligned}
$$

Although the variables $Y$ and $Z$ involve impulsive noise, the median difference of $|Y|-B$ and $|Z|-B$ works well on it.

\section{Proof of Property 4}

To prove Property 4, it is only necessary to prove that $\left|R_{w}^{\text {mdco }}\right|<\left|R_{w}^{\text {crco }}\right|$ when the sampling data contains impulsive noise. According to Property 3, The authors only need to prove $\left|R_{\mathrm{MDCO}}\right|<\left|R_{\mathrm{CRCO}}\right|$.

For the MDCO and CRCO algorithms, the authors have $\left|R_{\mathrm{MDCO}}\right|-\left|R_{\mathrm{CRCO}}\right|$

$$
\begin{aligned}
= & \mid E\left[\exp \left(-\frac{|| Y|-B|^{2}}{2 \sigma^{2}}\right) \exp \left(-\frac{|| Z|-B|^{2}}{2 \sigma^{2}}\right)\right. \\
& \left.\cdot \exp \left(-\frac{|Y-Z|^{2}}{2 \sigma^{2}}\right) Y Z\right]|-| E\left[\exp \left(-\frac{|Y-Z|^{2}}{2 \sigma^{2}}\right) Y Z\right] \mid \\
\approx & \mid E\left[\exp \left(-\frac{\left|W_{Y}\right|^{2}}{2 \sigma^{2}}\right) \exp \left(-\frac{\left|W_{Z}\right|^{2}}{2 \sigma^{2}}\right)\right. \\
& \left.\cdot \exp \left(-\frac{\left|W_{Y}-W_{Z}\right|^{2}}{2 \sigma^{2}}\right) Y Z\right] \mid \\
& -\left|E\left[\exp \left(-\frac{\left|W_{Y}-W_{Z}\right|^{2}}{2 \sigma^{2}}\right) Y Z\right]\right|
\end{aligned}
$$

For the generalized covariance of the two variables $Y$ and $Z$ at a given moment, (D.1) can be rewritten as

$$
\begin{aligned}
& \left|R_{\mathrm{MDCO}}\right|-\left|R_{\mathrm{CRCO}}\right| \\
& \approx E\left[\left(\exp \left(-\frac{\left|W_{Y}\right|^{2}}{2 \sigma^{2}}\right) \exp \left(-\frac{\left|W_{Z}\right|^{2}}{2 \sigma^{2}}\right)-1\right)\right. \\
& \left.\quad \cdot \exp \left(-\frac{\left|W_{Y}-W_{Z}\right|^{2}}{2 \sigma^{2}}\right)|Y Z|\right]
\end{aligned}
$$

When the variables $Y$ and $Z$ contain impulsive noise, the authors can get $\exp \left(-\left|W_{Y}\right|^{2} / 2 \sigma^{2}\right)<1$ and $\exp \left(-\left|W_{Z}\right|^{2} / 2 \sigma^{2}\right)<1$. Then, 


$$
\begin{aligned}
& \left|R_{\mathrm{MDCO}}\right|-\left|R_{\mathrm{CRCO}}\right| \\
& \approx E\left[\left(\exp \left(-\frac{\left|W_{Y}\right|^{2}}{2 \sigma^{2}}\right) \exp \left(-\frac{\left|W_{Z}\right|^{2}}{2 \sigma^{2}}\right)-1\right)\right. \\
& \left.\cdot \exp \left(-\frac{\left|W_{Y}-W_{Z}\right|^{2}}{2 \sigma^{2}}\right)|Y Z|\right]<0 .
\end{aligned}
$$

In summary, the MDCO algorithm performs better than the CRCO algorithm under impulsive noise environments, and results in $R_{\mathrm{MDCO}} \longrightarrow 0$.

\section{E. Proof of Property 5}

Under the premise of Property 4 , there exists $0<\eta<1$ which makes

$$
R_{\mathrm{MDCO}}=\eta R_{\mathrm{CRCO}}
$$

Meanwhile, $R_{\mathrm{CRCO}}$ is bounded with [24] and can be expressed as

$$
-\frac{\sigma^{3}}{\sqrt{2 \pi} \gamma} \leq R_{\mathrm{CRCO}} \leq\left[4 \frac{\Gamma(1) \Gamma(-1 / \alpha)}{\alpha \sqrt{\pi} \Gamma(-1 / 2)} \gamma^{1 / \alpha}\right]^{2} .
$$

Combining (E.3) with (E.2), the authors can easily get

$$
-\frac{\sigma^{3}}{\sqrt{2 \pi} \gamma} \leq \frac{R_{\mathrm{MDCO}}}{\eta} \leq\left[4 \frac{\Gamma(1) \Gamma(-1 / \alpha)}{\alpha \sqrt{\pi} \Gamma(-1 / 2)} \gamma^{1 / \alpha}\right]^{2} .
$$

Transforming (E.4), the authors have

$$
-\frac{\sigma^{3}}{\sqrt{2 \pi} \gamma} \eta \leq R_{\mathrm{MDCO}} \leq\left[4 \frac{\Gamma(1) \Gamma(-1 / \alpha)}{\alpha \sqrt{\pi} \Gamma(-1 / 2)} \gamma^{1 / \alpha}\right]^{2} \eta,
$$

which means that $R_{\mathrm{MDCO}}$ is bounded.

\section{Data Availability}

All the data generated or analyzed in this study are available from the corresponding author on reasonable request.

\section{Conflicts of Interest}

The authors declare that there are no conflicts of interest regarding the publication of this paper.

\section{Acknowledgments}

The paper was sponsored by National Key R\&D Program of China (no. 2017YFB0702300) and National Natural Science Foundation of China (no. 61971031).

\section{References}

[1] J. Zheng and M. Kaveh, "Sparse spatial spectral estimation: a covariance fitting algorithm, performance and regularization," IEEE Transactions on Signal Processing, vol. 61, no. 11, pp. 2767-2777, 2013.

[2] Q. Li, B. Liao, L. Huang, C. Guo, G. Liao, and S. Zhu, "A robust STAP method for airborne radar with array steering vector mismatch," Signal Processing, vol. 128, pp. 198-203, 2016.

[3] Z. Xiahou and X. Zhang, "Adaptive localization in wireless sensor network through Bayesian compressive sensing," International Journal of Distributed Sensor Networks, vol. 11, no. 8, 2015.

[4] F.-G. Yan, Y. Shen, and M. Jin, "Fast DOA estimation based on a split subspace decomposition on the array covariance matrix," Signal Processing, vol. 115, pp. 1-8, 2015.

[5] F. Ma and X. Zhang, "Wideband DOA estimation based on focusing signal subspace," Signal, Image and Video Processing, vol. 13, no. 4, pp. 675-682, 2019.

[6] M. R. Anbiyaei, W. Liu, and D. C. McLernon, "Performance improvement for wideband DOA estimation with white noise reduction based on uniform linear arrays," in Proceedings of the 2016 IEEE Sensor Array and Multichannel Signal Processing Workshop (SAM), pp. 1-5, IEEE, Rio de Janeiro, Brazil, July 2016.

[7] R. Schmidt, "Multiple emitter location and signal parameter estimation," IEEE Transactions on Antennas and Propagation, vol. 34, no. 3, pp. 276-280, 1986.

[8] C. Qian, L. Huang, and H. C. So, "Improved unitary rootMUSIC for DOA estimation based on pseudo-noise resampling," IEEE Signal Processing Letters, vol. 21, no. 2, pp. 140-144, 2014.

[9] R. Roy and T. Kailath, "ESPRIT-estimation of signal parameters via rotational invariance techniques," IEEE Transactions on Acoustics, Speech, and Signal Processing, vol. 37, no. 7, pp. 984-995, 1989.

[10] A. Hu, T. Lv, H. Gao, Z. Zhang, and S. Yang, "An ESPRITbased approach for 2-D localization of incoherently distributed sources in massive MIMO systems," IEEE Journal of Selected Topics in Signal Processing, vol. 8, no. 5, pp. 996-1011, 2014.

[11] L. Lu and H. Zhao, "Active impulsive noise control using maximum correntropy with adaptive kernel size," Mechanical Systems and Signal Processing, vol. 87, pp. 180-191, 2017.

[12] W.-J. Zeng, H. C. So, and L. Huang, "p-MUSIC: robust direction-of-arrival estimator for impulsive noise environments," IEEE Transactions on Signal Processing, vol. 61, no. 17, pp. 4296-4308, 2013.

[13] P. Wang, T.-S. Qiu, F.-Q. Ren, and A.-M. Song, "A robust DOA estimator based on the correntropy in alpha-stable noise environments," Digital Signal Processing, vol. 60, pp. 242-251, 2017.

[14] J.-F. Zhang and T.-S. Qiu, "A robust correntropy based subspace tracking algorithm in impulsive noise environments," Digital Signal Processing, vol. 62, pp. 168-175, 2017.

[15] Z. Chen, X. Geng, and F. Yin, "A harmonic suppression method based on fractional lower order statistics for power system," IEEE Transactions on Industrial Electronics, vol. 63, no. 6, pp. 3745-3755, 2016.

[16] X. Jiang, W.-J. Zeng, A. Yasotharan, H. C. So, and T. Kirubarajan, "Minimum dispersion beamforming for nonGaussian signals," IEEE Transactions on Signal Processing, vol. 62, no. 7, pp. 1879-1893, 2014

[17] P. Tsakalides and C. L. Nikias, "The robust covariation-based MUSIC (ROC-MUSIC) algorithm for bearing estimation in impulsive noise environments," IEEE Transactions on Signal Processing, vol. 44, no. 7, pp. 1623-1633, 1996.

[18] T.-H. Liu and J. M. Mendel, "A subspace-based direction finding algorithm using fractional lower order statistics," IEEE Transactions on Signal Processing, vol. 49, no. 8, pp. 1605-1613, 2001. 
[19] H. Belkacemi and S. Marcos, "Robust subspace-based algorithms for joint angle/doppler estimation in non-Gaussian clutter," Signal Processing, vol. 87, no. 7, pp. 1547-1558, 2007.

[20] I. Santamaria, P. P. Pokharel, and J. C. Principe, "Generalized correlation function: definition, properties, and application to blind equalization," IEEE Transactions on Signal Processing, vol. 54, no. 6, pp. 2187-2197, 2006.

[21] B. Chen and J. C. Principe, "Maximum correntropy estimation is a smoothed MAP estimation," IEEE Signal Processing Letters, vol. 19, no. 8, pp. 491-494, 2012.

[22] W. Ma, B. Chen, J. Duan, and H. Zhao, "Diffusion maximum correntropy criterion algorithms for robust distributed estimation," Digital Signal Processing, vol. 58, pp. 10-19, 2016.

[23] Z. Jinfeng and Q. Tianshuang, "A novel covariation based noncircular sources direction finding method under impulsive noise environments," Signal Processing, vol. 98, pp. 252-262, 2014.

[24] J. Zhang, T. Qiu, A. Song, and H. Tang, "A novel correntropy based DOA estimation algorithm in impulsive noise environments," Signal Processing, vol. 104, pp. 346-357, 2014.

[25] R. Patel, M. P. Janawadkar, S. Sengottuvel, K. Gireesan, and T. S. Radhakrishnan, "Effective extraction of visual eventrelated pattern by combining template matching with ensemble empirical mode decomposition," IEEE Sensors Journal, vol. 17, no. 7, pp. 2146-2153, 2017.

[26] W. Liu, P. P. Pokharel, and J. C. Principe, "Correntropy: properties and applications in non-Gaussian signal processing," IEEE Transactions on Signal Processing, vol. 55, no. 11, pp. 5286-5298, 2007.

[27] N. Erdol, C. Castelluccia, and A. Zilouchian, "Recovery of missing speech packets using the short-time energy and zerocrossing measurements," IEEE Transactions on Speech and Audio Processing, vol. 1, no. 3, pp. 295-303, 1993.

[28] T. Dai, W. Lu, W. Wang, J. Wang, and S.-T. Xia, "Entropybased bilateral filtering with a new range kernel," Signal Processing, vol. 137, pp. 223-234, 2017.

[29] M. Zhang and B. K. Gunturk, "Multiresolution bilateral filtering for image denoising," IEEE Transactions on Image Processing, vol. 17, no. 12, pp. 2324-2333, 2008.

[30] I. Frosioa, K. Egiazarian, and K. Pulli, "Machine learning for adaptive bilateral filtering," in Proceedings of the Image Processing: Algorithms and Systems XIII, San Francisco, CA, USA, February 2015

[31] R. J. Kozick and B. M. Sadler, "Maximum-likelihood array processing in non-Gaussian noise with Gaussian mixtures," IEEE Transactions on Signal Processing, vol. 48, no. 12, pp. 3520-3535, 2000.

[32] B. D. Rao and K. V. S. Hari, "Performance analysis of rootMUSIC," IEEE Transactions on Acoustics, Speech, and Signal Processing, vol. 37, no. 12, pp. 1939-1949, 1989. 


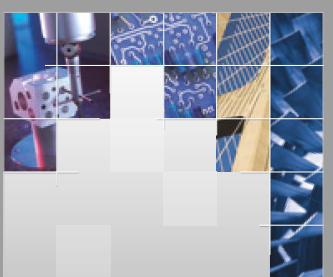

\section{Enfincering}
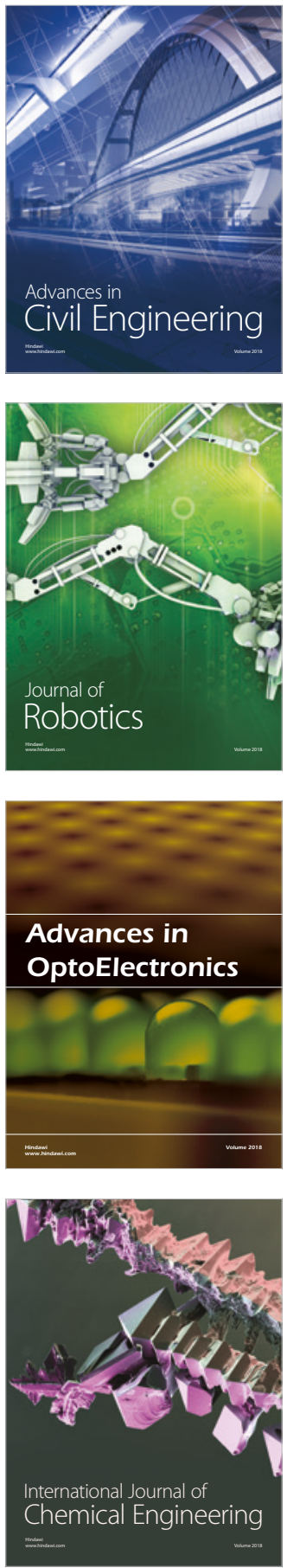

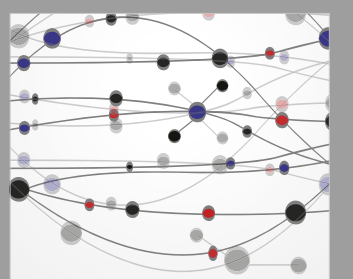

\section{Rotating \\ Machinery}

The Scientific World Journal

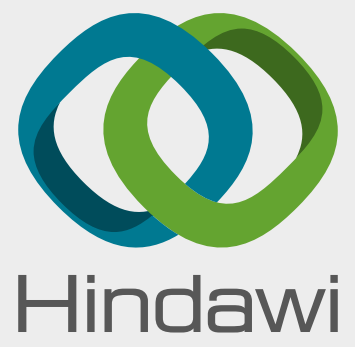

Submit your manuscripts at

www.hindawi.com
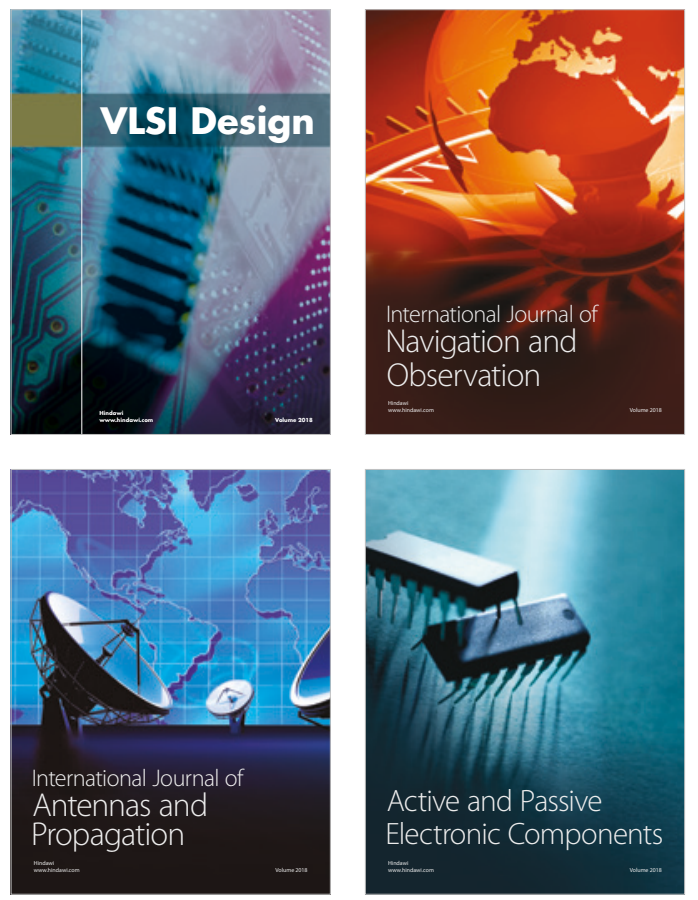
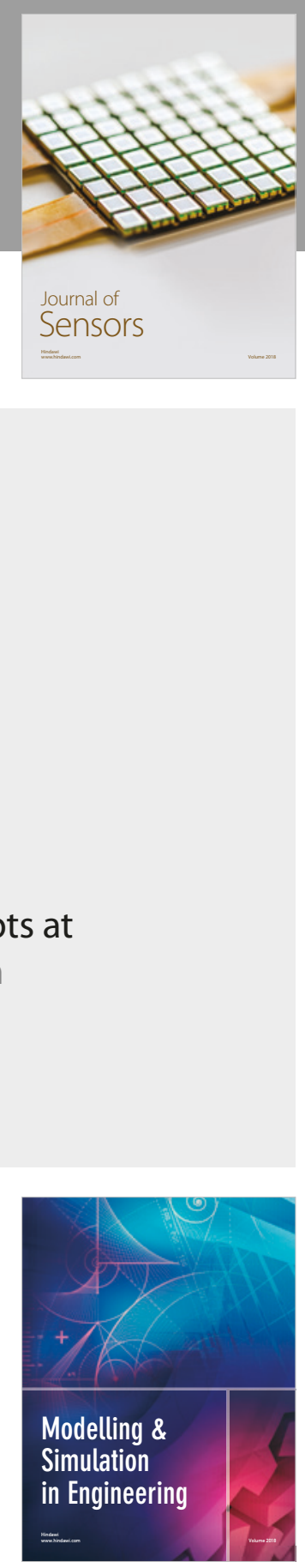

\section{Advances \\ Multimedia}
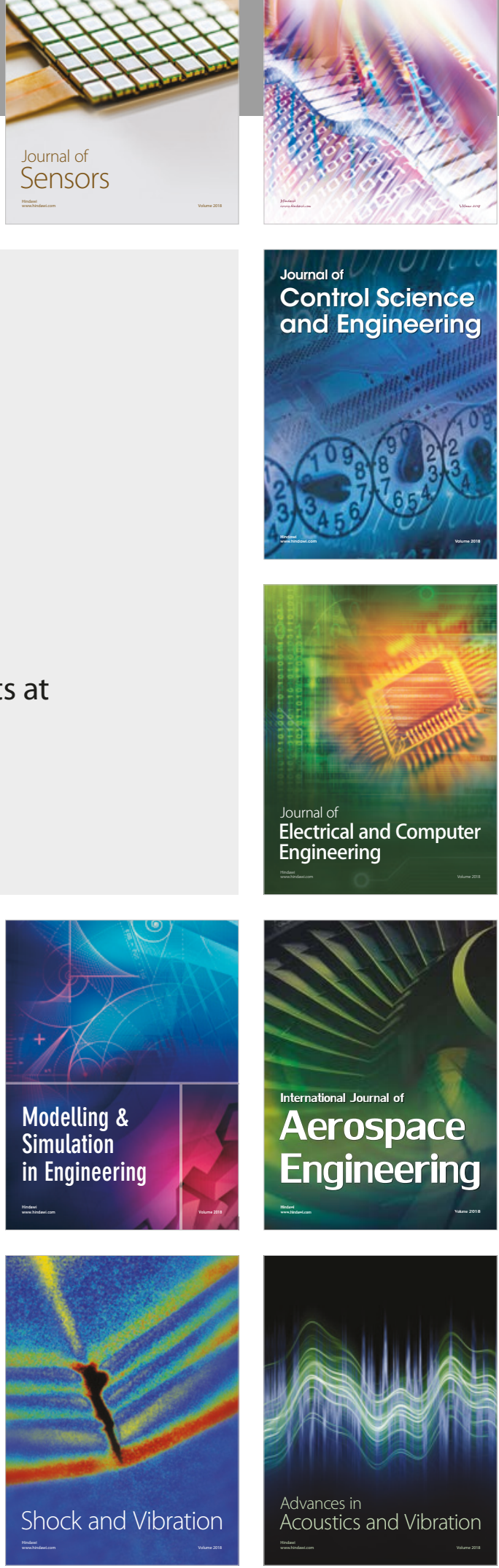